\title{
Influencia de características microestructurales de caolinita en las propiedades de sus pastas de colaje
}

\author{
C. GARCÍA-PORTILLO *; J. BASTIDA **; P. PARDO **; G. RODRÍGUEZ-LÓPEZ*; $M^{\text {a }}$ J. LACRUZ*; Ma L. VILAR *; A. LÁZARO** \\ * Escuela Superior de Cerámica de Manises. C/ Ceramista Alfons Blat, 22, 45940 Manises (Valencia) \\ ** Departamento de Geología. Universidad de Valencia. C/ Dr. Moliner, 50. 46100 Burjasot (Valencia)
}

\begin{abstract}
En el presente trabajo se realiza la caracterización granulométrica, mineralógica, química y microestructural de 9 caolines y 4 arcillas de amplio uso en el clúster cerámico de Manises. Se determinan propiedades en crudo (viscosidad, velocidad de formación de capa) y en cocido (absorción de agua, contracción, densidad aparente) y se analizan las relaciones existentes entre dichas propiedades y las características composicionales, prestándose especial atención a la influencia de la microestructura de la caolinita. Se concluye que existe relación entre el tamaño de cristalito de la caolinita y propiedades en cocido como la absorción de agua y la densidad aparente. Destaca como principal parámetro de composición el contenido en fracción fina de los materiales, que se relaciona con la densidad aparente, la absorción de agua, y la velocidad de formación de pared.
\end{abstract}

Palabras clave: Arcillas, Caolinita, Difracción de Rayos X, Tamaño de Cristalito

Microstructure of kaolinite and technological properties of kaolinitic casting clays.

The granulometrical, chemical, mineralogical and microestructural characterizations of 9 kaolins and 4 clays widely used as raw materials at the Manises ceramic cluster were performed. Technological properties such as viscosity, thickness formation speed, shrinkage, water absorption and apparent density of all materials were studied. The aim of the work is to analyse the relationships between the characteristics of the materials and their technological properties, paying special attention to the kaolinite crystallite size. Significant relationships between kaolinite crystallite size and properties after firing such as water absorption and apparent density were found. The most important characteristic was the clay fraction, showing relationship with water absorption, apparent density and casting rate.

Keywords: Clays, Kaolinite, X-Ray Diffraction, Crystallite - size.

\section{INTRODUCCIÓN Y OBJETIVO}

Las materias primas ricas en caolinita son ampliamente utilizadas industrialmente; Konta (1), resume un estudio sobre dichas aplicaciones, que incluyen cargas (en papel, gomas, plásticos), cerámicas (incluyendo refractarios, cementos especiales y fabricación de fibras de vidrio y sialones) catalíticas (en la industria química, principalmente caolinita activada) y otras (tintes, pinturas, adhesivos, adsorbentes). Propiedades importantes para distintas aplicaciones son: blancura, blandura, actividad superficial y propiedades adsorbentes y reológicas. En el caso de las pastas para colaje las propiedades reológicas son críticas. Según Williams y col. (2) el comportamiento reológico de las suspensiones de caolín está gobernado fundamentalmente por las propiedades de las partículas de caolinita: tamaño y distribución de partícula, morfología, agregación, carga superficial e impurezas químicas y mineralógicas. Las diferencias en estas propiedades controlan el comportamiento de una suspensión de caolinita frente a cambios de $\mathrm{pH}$, temperatura y composición química del agua.

La caolinita es un filosilicato con marcada exfoliación basal, que presenta láminas con prevalencia de las formas $\{001\}$. Pueden producirse láminas más finas mediante delaminación continuada a lo largo de los planos (001), de manera que el caolín consta de una débil agregación de apilamientos de láminas de caolinita con orientación aleatoria, paquetes más pequeños, manojos y láminas individuales.
Pueden presentarse unidades más grandes (llamadas libros, acordeones o gusanos) ligeramente expandidas si durante el crecimiento cristalino había disponibilidad de espacio suficiente.

La determinación de tamaños de cristalito en caolinita a través de la fórmula de Scherrer se llevó a cabo en (2). Los procedimientos de Williamson y Hall (3) y Warren y Averbach (4) fueron empleados por Amigó y col. (5) para el análisis de las reflexiones 001 de la caolinita.

El uso del valor de la anchura a media altura (FWHM) para la reflexión (001) como un "índice de cristalinidad" fue propuesto por Amigó y col. (5) y fue comparado con otros procedimientos de difracción de rayos X (DRX) por Aparicio y Galán (6).

Un estudio reciente acerca de la cristalinidad de caolinita (ver las observaciones de Guggenheim (7) para el uso de este término) mediante métodos de DRX ha sido realizado por Clausell (8), en el cual se aplican los métodos de Williamson y Hall, Warren y Averbach y el descrito por Eberl y col. (9), a caolinitas de caolines del Macizo Ibérico.

En el presente trabajo se determina mediante difracción de rayos X características microestructurales en la caolinita, relativas a la dirección [001]. Así mismo se realizan análisis de distribución de tamaño de partícula y de composición mineralógica de los distintos materiales, completando el estudio con la evaluación de la viscosidad y velocidad de 
colaje de las respectivas pastas, parámetros tecnológicamente importantes y ampliamente empleados en la industria para determinar el comportamiento en crudo de las pastas. Para evaluar las propiedades tras la cocción se determinaron la densidad aparente, la contracción y la absorción de agua de los materiales.

El objetivo de este trabajo es relacionar las características microestructurales, de composición, química y granulométrica de las materias primas con sus propiedades en crudo y tras la cocción.

\section{MATERIALES}

Para el presente trabajo se han estudiado 9 caolines $(k 1, \ldots$, k9) y 4 arcillas (a1,..., a4). El caolín 1 procede de Cornualles, Inglaterra, los caolines k2 y k3 de Valencia; los k4, k5, k6, k7 de Guadalajara, el k8 de Lugo y el k9 de Cuenca. Los caolines en cuestión son caolines elaborados con caolines brutos del tipo I de Galán y Espinosa (10), salvo el k8, cuya materia prima corresponde al tipo IV de la referida clasificación.

Las arcillas a1, a3 y a4 proceden de la zona de Devon (Reino Unido), y la a2 es una arcilla refractaria, procedente de Francia.

Todos los materiales estudiados son materias primas industriales, ampliamente utilizados por las empresas del clúster cerámico de Manises.

En el apartado 6 se presentan los resultados de los ensayos aplicados a los materiales.

\section{MÉTODOS EXPERIMENTALES}

\subsection{Preparación de la barbotina}

Se tomaron $500 \mathrm{~g}$ del material con un contenido en humedad comprendido entre el $0.5-2 \%$, y se pasaron por un tamiz de $0.5 \mu \mathrm{m}$.

La muestra ya tamizada se suspendió en agua, y posteriormente se añadió el defloculante $\left(\mathrm{Na}_{2} \mathrm{SiO}_{3}\right)$ en etapas de $0.2 \mathrm{ml}$, agitando durante $5 \mathrm{~min}$, efectuándose adiciones hasta dejar de apreciarse mejora en el valor de viscosidad.

\subsection{Determinación de propiedades de procesado}

La determinación de la viscosidad se realizó con un viscosímetro Gallenkamp, como se describe en Galán y Espinosa (10).

Una vez puesta la barbotina en las condiciones de densidad, viscosidad y tixotropía adecuadas, se realizaron coladas a distintos tiempos $(5,9$ y 12 minutos) en moldes de idénticas características.

Una vez desmoldadas y secas las probetas, se mide el espesor en distintos puntos. Se representan los cuadrados de los valores medios obtenidos respecto al tiempo de colada, obteniéndose una recta cuya pendiente da el valor de la velocidad de formación de capa, ver Galán y Espinosa (10).

\subsection{Determinación de propiedades del producto cocido}

Se determinó la absorción de agua y la densidad aparente según la norma UNE-EN ISO 10545-3 (11), y la contracción lineal según el procedimiento E-9 de Amorós y col. (12) para temperaturas de cocción de 1100, 1150, 1200, 1240 y $1280^{\circ} \mathrm{C}$.
Las mayores diferencias entre los resultados de estos ensayos sobre los materiales se dieron para $1200{ }^{\circ} \mathrm{C}$, recogiéndose dichos valores en la tabla IV.

\subsection{Análisis por difracción de rayos X (DRX)}

Mediante difracción de rayos X de polvo se determinaron las composiciones mineralógicas y se efectuaron asimismo determinaciones del tamaño de cristalito en caolinita. Los registros se efectuaron en un difractómetro Bruker D-5000, trabajando a $40 \mathrm{kV}$ y $30 \mathrm{~mA}$, con ventanas de divergencia de $1^{\circ}$ y antiscatering de $1^{\circ}$.

Para la identificación de los filosilicatos y el análisis semicuantitativo se emplearon los métodos de Warshaw y Roy (13) y Davis y Smith (14) y (15). Las condiciones empleadas para estos registros se resumen en la tabla I. La adquisición y el tratamiento de datos para identificación se efectuaron utilizando diferentes programas del paquete Diffrac-Plus, suministrado por Bruker AXS.

TABLA I. CONDICIONES DE LOS REGISTROS DE DRX. (A.O. = AGREGADOS ORIENTADOS)

\begin{tabular}{|lccc|}
\hline \multicolumn{1}{|c}{ Registro } & Paso $\left({ }^{\mathbf{o}} \mathbf{2} \theta\right)$ & Tiempo $(\mathbf{s})$ & Intervalo $\left({ }^{\mathbf{0}} \mathbf{2} \theta\right)$ \\
\hline Polvo cristalino & 0.04 & 4 & $2-72$ \\
(A.O.) Normal & 0.02 & 3 & $2-32$ \\
(A.O.) Calentado & 0.04 & 3 & $2-22$ \\
(A.O.) Glicolado & 0.04 & 3 & $2-17$ \\
\hline
\end{tabular}

El tratamiento de los datos de difracción para obtención de los perfiles utilizados en análisis microestructural se efectuó utilizando el programa Profile (de Secabim, suministrado por Bruker AXS). El método de análisis utilizado ha sido el de la función de Voigt, introducido por Langford (16) y basado en que el perfil de un pico de difracción puede describirse en primera aproximación por la convolución de una curva de Cauchy (Lorente) y una curva de Gauss (la convolución de ambas es la denominada función de Voigt).

Las dos principales contribuciones microestructurales al ensanchamiento de los picos de difracción, tamaño de los cristalitos y distorsiones, originan perfiles distintos. Así, los efectos de tamaño producen una distribución de intensidades de tipo Lorentziano, mientras que las distorsiones dan lugar a una distribución gaussiana, como expuso Langford (16). La proporción de las contribuciones de Gauss y Cauchy al perfil de Voigt pueden expresarse empleando la anchura integral " $\beta$ " de los picos de difracción, con lo que obtenemos:

$$
\beta_{\mathrm{G}} / \beta \text { (Gauss) } \quad \beta_{\mathrm{C}} / \beta \text { (Cauchy) }
$$

siendo $\beta_{\mathrm{G}}$ y $\beta_{\mathrm{C}}$ las anchuras integrales de perfil gaussiano y lorentziano respectivamente, según las relaciones del análisis de Voigt, obtenidas empíricamente, tal y como se recogen, por ejemplo, en el trabajo de Keijser y col. (17).

Una vez conocidas $\beta_{\mathrm{G}}$ y $\beta_{\mathrm{C}}$ se procede a obtener por deconvolución las componentes de Gauss y Cauchy del perfil de difracción puro. Para ello se emplean las conocidas fórmulas de corrección:

$$
\begin{array}{ll}
\beta_{\mathrm{C}}{ }^{\mathrm{f}}=\beta_{\mathrm{C}}{ }^{\mathrm{h}}-\beta_{\mathrm{C}}^{\mathrm{g}} & \text { (para un perfil de Cauchy) } \\
\left(\beta_{\mathrm{G}}{ }^{\mathrm{f}}{ }^{2}=\left(\beta_{\mathrm{G}}{ }^{\mathrm{h}}\right)^{2}-\left(\beta_{\mathrm{G}}^{\mathrm{g}}\right)^{2}\right. & \text { (para un perfil de Gauss) }
\end{array}
$$


donde " $\mathrm{f}$ " se refiere al perfil de difracción puro, " $\mathrm{h}$ " al experimental y " $\mathrm{g}$ " al instrumental.

Se considera que el efecto del tamaño de los cristalitos en el ensanchamiento se encuentra enteramente representado por la componente de Cauchy del perfil puro, mientras que la contribución de las distorsiones queda recogida en la componente de Gauss. El tamaño aparente de los cristalitos se obtiene por:

$$
<\mathrm{Dv}>=\lambda / \beta_{\mathrm{C}}{ }^{\mathrm{f}} \cos \theta
$$

y el parámetro de distorsión según:

$$
\mathrm{e}=\beta_{\mathrm{C}}^{\mathrm{f}} / 4 \operatorname{tg} \theta
$$

siendo $\lambda$ la longitud de onda empleada $(\AA ̊)$ y $\theta$ el ángulo de Bragg en radianes. En ambos casos $\beta_{\mathrm{C}}{ }^{\mathrm{f}}$ se expresa en la escala $2 \theta$.

\section{RESULTADOS Y DISCUSIÓN}

La tabla II recoge los datos de composición química y mineralógica. Los datos de composición química proceden de la hoja técnica de producto suministrada por el proveedor. Las arcillas se caracterizan por valores superiores, en todos los casos, para $\mathrm{Fe}_{2} \mathrm{O}_{3}, \mathrm{TiO}_{2}, \mathrm{CaO}, \mathrm{MgO}$, y $\mathrm{Na}_{2} \mathrm{O}$.

Los caolines se encuentran dentro de los límites composicionales que establece la EKA (European Kaolin Association) en su definición composicional de caolín, mientras que todas las arcillas consideradas estarían fuera de

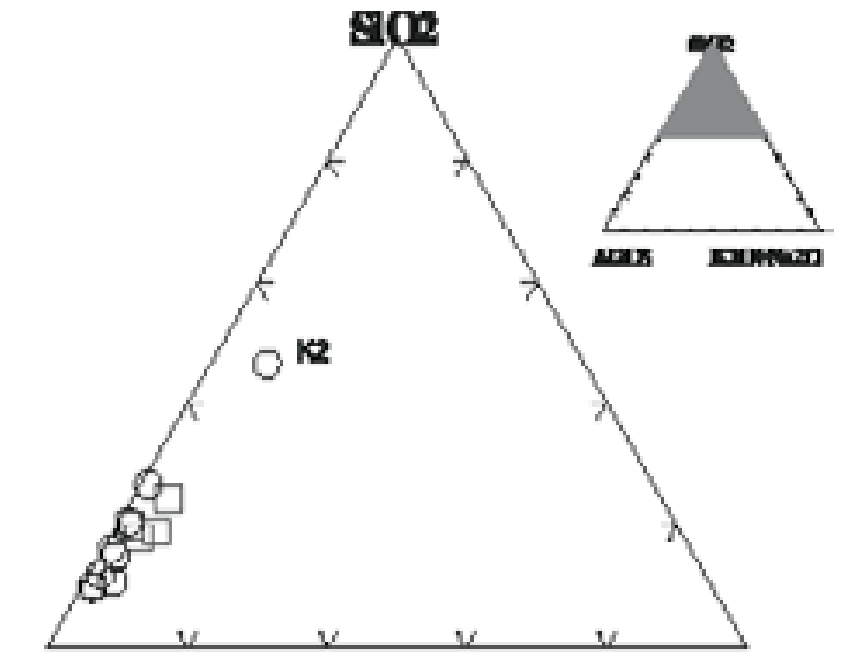

Al30B

No+N20

Fig. 1.- Ubicación de las muestras en un diagrama de composición

equivalente) tratada con etilenglicol. Se observa que los constituyentes presentes en todos los casos son caolinita y minerales micáceos ("illita"); en el caso de k1 se aprecia también presencia de esmectitas, que se hacen patentes por la presencia de espaciados de $\approx 17 \AA \mathrm{y} \approx 19 \AA ̊$ en el agregado orientado etilenglicolado.

La figura 3 muestra los registros de agregados etilenglicolados de las muestras con valores extremos de tamaño de cristalito. Debe notarse que precisamente la

\begin{tabular}{|c|c|c|c|c|c|c|c|c|c|c|c|c|c|}
\hline & \multicolumn{9}{|c|}{ Análisis Químico (\%) } & \multicolumn{4}{|c|}{ Análisis Mineralógico (\%) } \\
\hline & $\mathrm{SiO}_{2}$ & $\mathrm{Al}_{2} \mathrm{O}_{3}$ & $\mathrm{Fe}_{2} \mathrm{O}_{3}$ & $\mathrm{TiO}_{2}$ & $\mathrm{CaO}$ & $\mathrm{MgO}$ & $\mathrm{K}_{2} \mathrm{O}$ & $\mathrm{Na}_{2} \mathrm{O}$ & P.F. & $\mathbf{Q}$ & $\mathbf{K}$ & I & $\mathrm{Fd}$ \\
\hline a1 & 49.00 & 31.00 & 1.20 & 0.90 & 0.20 & 0.30 & 2.10 & 0.30 & 14.50 & 42.0 & 53.5 & 2.2 & 2.3 \\
\hline a3 & 50.00 & 32.90 & 1.20 & 1.00 & 0.20 & 0.30 & 1.60 & 0.20 & 12.60 & 81.3 & 12.5 & 1.1 & 5.1 \\
\hline a4 & 54.10 & 30.80 & 1.00 & 1.20 & 0.20 & 0.30 & 2.20 & 0.10 & 10.20 & 69.0 & 26.6 & 1.7 & 2.6 \\
\hline k1 & 48.00 & 37.00 & 0.70 & 0.02 & 0.07 & 0.30 & 1.70 & 0.10 & 12.50 & 48.9 & 45.6 & 0.7 & 4.7 \\
\hline k2 & 67.68 & 21.00 & 0.21 & 0.23 & 0.20 & 0.24 & 3.67 & 0.10 & 6.51 & 15.2 & 81.7 & 0.9 & 2.2 \\
\hline k3 & 50.34 & 35.89 & 0.35 & 0.13 & 0.12 & 0.14 & 0.47 & 0.01 & 12.45 & 4.4 & 90.2 & 2.6 & 2.7 \\
\hline k4 & 47.62 & 38.20 & 0.50 & 0.11 & 0.07 & 0.06 & 0.60 & 0.04 & 12.80 & 38.8 & 49.3 & 2.1 & 9.9 \\
\hline k5 & 55.12 & 31.37 & 0.63 & 0.23 & 0.11 & 0.16 & 0.36 & 0.07 & 11.61 & 10.9 & 86.6 & 0.8 & 1.7 \\
\hline k6 & 47.50 & 38.20 & 0.45 & 0.11 & 0.07 & 0.05 & 0.55 & 0.05 & 13.00 & 5.6 & 93.2 & 0.8 & 0.4 \\
\hline k7 & 48.40 & 37.50 & 0.48 & 0.13 & 0.09 & 0.06 & 0.70 & 0.05 & 12.58 & 8.9 & 87.8 & 0.9 & 2.4 \\
\hline k9 & 52.30 & 33.90 & 0.76 & 0.20 & 0.24 & - & 0.45 & 0.10 & 11.40 & 42.8 & 53.7 & 1.8 & 1.7 \\
\hline
\end{tabular}

TABLA II. ANÁLISIS QUÍMICO Y MINERALÓGICO DE LOS MATERIALES ESTUDIADOS. (Q, CUARZO; K, CAOLINITA; I, ILLITA; FD, FELDESPATOS).

tal definición para $\mathrm{Fe}_{2} \mathrm{O}_{3^{\prime}} \mathrm{TiO}_{2}, \mathrm{MgO}$, y $\mathrm{Na}_{2} \mathrm{O}$, y la a1, además para la pérdida al fuego $(\mathrm{PF})$.

Dentro de los caolines destacan contenidos relativamente elevados en $\mathrm{MgO}$ para las muestras $\mathrm{k} 1 \mathrm{y}$ k2.

Desde el punto de vista de aplicación a porcelanas, la consideración de la composición química relativa a $\mathrm{SiO}_{2^{\prime}}$ $\mathrm{Al}_{2} \mathrm{O}_{3}$ y $\mathrm{Na}_{2} \mathrm{O}+\mathrm{K}_{2} \mathrm{O}$, pone de manifiesto una elevada similitud composicional que puede apreciarse en la figura 1.

La figura 2 presenta los registros difractométricos de la fracción arcillosa (menor de $2 \mu \mathrm{m}$ de diámetro esférico muestra k1, de mayor tamaño de cristalito, presenta cuantías significativas de esmectita, hecho que se relaciona con el elevado valor de $\mathrm{MgO}$ apreciado en el análisis químico de esta muestra con respecto al resto de caolines.

La tabla III recoge por una parte, las fracciones de volúmenes más significativas de las distribuciones granulométricas de los materiales, y por otra, los parámetros microestructurales (tamaño de cristalito $(<\mathrm{Dv}>$ ) y distorsión (e)) obtenidos para la caolinita en la dirección [001] de los materiales estudiados.

La figura 4 presenta las distribuciones granulométricas 


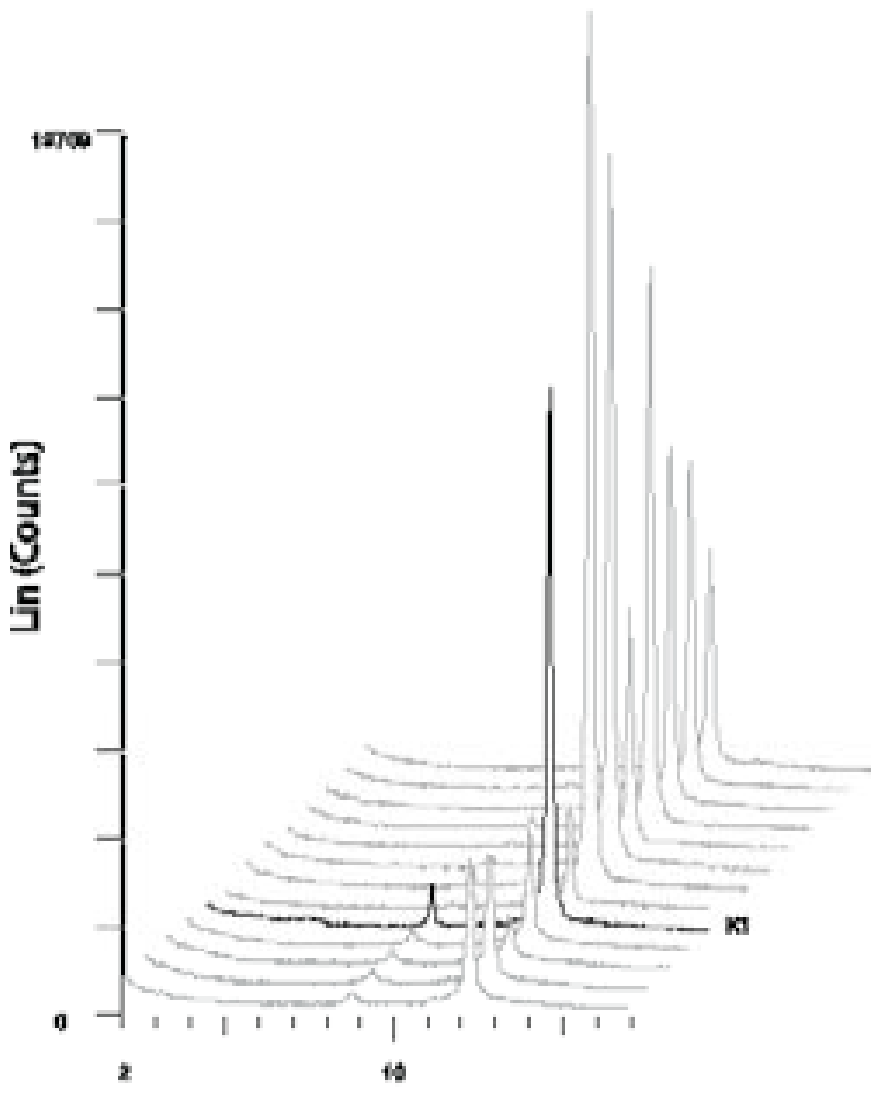

\section{2-Theta - Scale}

Fig. 2.- Registros de difracción de los agregados etilenglicolados de todas las muestras.

de los materiales, y pone de manifiesto con claridad el desplazamiento a menores tamaños de la media de la distribución en las arcillas. La tabla IV recoge las propiedades tecnológicas en crudo y en cocido consideradas. Los valores de contracción, densidad aparente y absorción de agua reflejan que, como observó Gippini (18), cualquiera que sea la vía por la que se lleve a cabo la sinterización, esta va acompañada de una disminución de volumen, que se realiza a expensas de los poros interiores y exteriores, abiertos o cerrados, lo que supone una densificación del material y una disminución de su absorción de agua.

Para establecer relaciones entre las características composicionales y las propiedades tecnológicas (de procesado y en cocido) se ha practicado un análisis estadístico de correlaciones bivariadas; si se consideran todas las muestras las correlaciones son bajas; sin embargo la situación mejora si se prescinde de la muestra $k 1$, que presenta un comportamiento anómalo que asociamos a la presencia de cantidades apreciables de esmectitas.

Para valorar la influencia del tamaño de cristalito de la caolinita (frente a otras características composicionales) sobre las características tecnológicas consideradas se han comparado los coeficientes de correlación de Pearson.

En la tabla V se presentan las correlaciones más significativas entre las características tecnológicas y las propiedades de los materiales. La granulometría es la propiedad que presenta mayor influencia en la mayoría de características tecnológicas. Ello se traduce en mayores contracciones y densidades aparentes en cocido, para pastas obtenidas con arcillas (frente

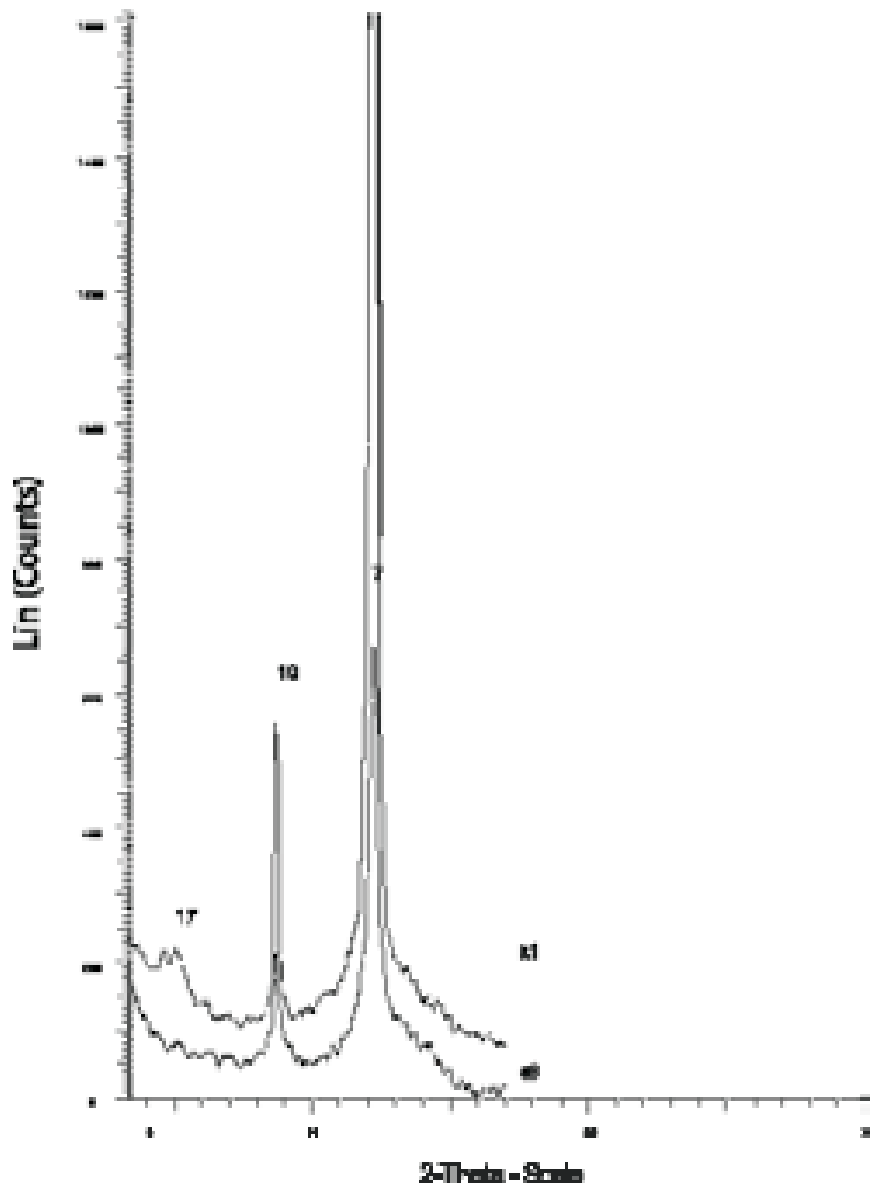

Fig. 3.- Registros de difracción de los agregados etilenglicolados de las muestras de cristalinidad extrema, $\mathrm{k} 1 \mathrm{y}$ a3.

a caolines). El porcentaje de partículas inferior a 2 y $6 \mu \mathrm{m}$ presenta una elevada correlación negativa con la velocidad de formación de capa y la absorción de agua, y positiva con la densidad aparente. Lo dicho está de acuerdo con lo observado por Caballero et al (19) y Ferrer et al (20) en sus trabajos sobre el efecto de la granulometría de las materias primas en la gresificación de diferentes productos cerámicos.

TABLA III. CARACTERÍSTICAS GRANULOMÉTRICAS DE LOS MATERIALES Y MICROESTRUCTURALES DE LA CAOLINITA. ( $<$ DV $>$, E, TAMAÑO DE CRISTALITO $Y$ DISTORSIÓN SEGÚN MÉTODO DE LA FUNCIÓN DE VOIGT)

\begin{tabular}{|c|c|c|c|c|}
\hline & \multicolumn{2}{|c|}{ Granulometría } & \multicolumn{2}{|c|}{ Análisis microestructural } \\
\hline & $\%<2.011 \mu$ & $\%<6.158 \mu$ & $<\mathrm{Dv}>()$ & e \\
\hline a1 & 29.8 & 67.7 & 232.6 & 0.0052 \\
\hline a2 & 29.9 & 64.2 & 216.1 & 0.0057 \\
\hline a3 & 31.0 & 66.5 & 161.9 & 0.0084 \\
\hline a4 & 33.1 & 71.7 & 230.6 & 0.0053 \\
\hline k1 & 20.4 & 58.1 & 313.4 & 0.0030 \\
\hline k2 & 22.9 & 44.7 & 267.5 & 0.0040 \\
\hline k3 & 9.0 & 31.9 & 295.9 & 0.0035 \\
\hline k4 & 18.4 & 55.5 & 272.0 & 0.0039 \\
\hline k5 & 20.8 & 51.0 & 279.7 & 0.0038 \\
\hline k6 & 16.5 & 48.3 & 275.4 & 0.0040 \\
\hline k7 & 14.4 & 41.5 & 271.4 & 0.0041 \\
\hline k8 & 15.3 & 46.8 & 280.2 & 0.0036 \\
\hline k9 & 19.8 & 49.2 & 258.1 & 0.0044 \\
\hline
\end{tabular}



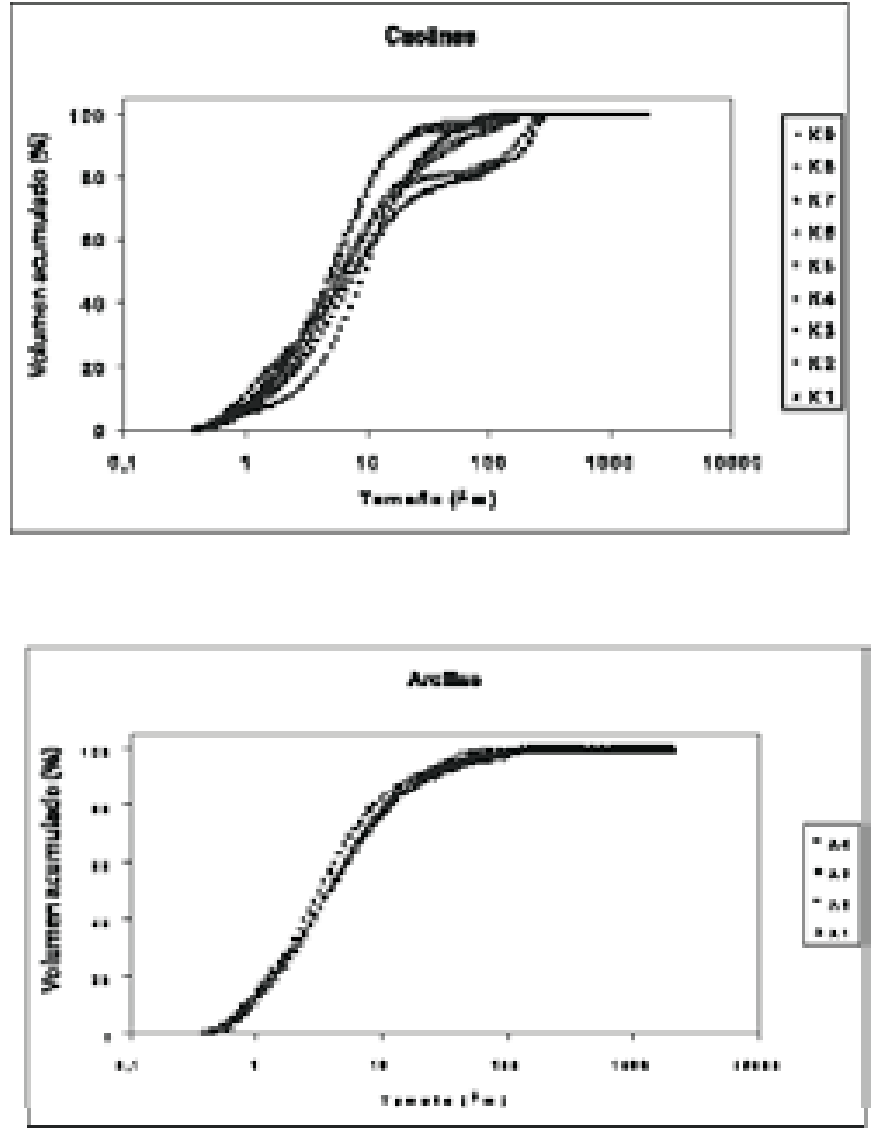

Fig. 4.- Análisis granulométrico de los materiales estudiados

TABLA IV. CARACTERÍSTICAS TECNOLÓGICAS EVALUADAS DE LOS MATERIALES

\begin{tabular}{|l|ccccc|}
\hline & $\begin{array}{c}\text { Vel. F.P. } \\
\left(\mathbf{m m}^{2} / \mathbf{m i n}\right)\end{array}$ & $\begin{array}{c}\text { Viscosidad } \\
\mathbf{(}^{\circ} \text { Gall) }\end{array}$ & $\begin{array}{c}\text { Contracción } \\
\mathbf{1 2 0 0}^{\mathbf{0}} \mathbf{( \% )}\end{array}$ & $\begin{array}{c}\text { Dens. Ap. } \\
\mathbf{1 2 0 0}^{\mathbf{0}} \mathbf{( g / \mathbf { c m } ^ { 3 } )}\end{array}$ & $\begin{array}{c}\text { Abs. Agua } \\
\mathbf{1 2 0 0}^{\circ} \mathbf{( \% )}\end{array}$ \\
\hline $\mathbf{a 1}$ & 0.0052 & 49 & 7.6 & 2.04 & - \\
$\mathbf{a 2}$ & 0.0048 & 25 & 7.5 & 2.01 & 1.53 \\
$\mathbf{a 3}$ & 0.0055 & 41 & 10.36 & 2.13 & 0.19 \\
$\mathbf{a 4}$ & 0.0070 & 60 & 9.96 & 2.13 & 0.23 \\
$\mathbf{k} \mathbf{1}$ & 0.0034 & 70 & 8.89 & 2.02 & 5.13 \\
$\mathbf{k 2}$ & 0.0049 & 26 & 7.6 & 1.66 & 19.23 \\
$\mathbf{k} 3$ & 0.0455 & 26 & 7.5 & 1.52 & 25.27 \\
$\mathbf{k} 4$ & 0.0076 & 8 & 3.82 & 1.81 & 15.94 \\
$\mathbf{k} 5$ & 0.0054 & 20 & 7.64 & 2.03 & 8.63 \\
$\mathbf{k 6}$ & 0.0084 & 30 & 4.1 & 1.6 & 23.09 \\
$\mathbf{k} 7$ & 0.0210 & 25 & 3 & 1.51 & 22.25 \\
$\mathbf{k} 8$ & 0.0092 & 7 & 5.2 & 1.71 & 21.49 \\
$\mathbf{k 9}$ & 0.0028 & 45 & 6.74 & 1.89 & 10.49 \\
\hline
\end{tabular}

Según los autores, mayores contenidos de fracción fina de una determinada materia prima aumentan la velocidad de sinterización. En la reaccionabilidad de materias arcillosas, sin la intervención de otras materias primas que generen fase liquida, prevalecen como mecanismo de la reaccionabilidad del material las reacciones sólido-sólido. En estas reacciones cobra mucha importancia la disminución del tamaño de partícula (elevada superficie específica) que facilitará el contacto entre las partículas, aumentando la reaccionabilidad física y química durante el proceso de cocción, como se describe en (21).
TABLA V. CORRELACIONES ENTRE CARACTERÍSTICAS TECNOLÓGICAS Y COMPOSICIONALES

\begin{tabular}{|c|c|c|c|}
\hline $\begin{array}{l}\text { Característica } \\
\text { tecnológica (c1) }\end{array}$ & $\begin{array}{c}\text { Característica } \\
\text { composicional (c2) }\end{array}$ & $\begin{array}{c}\text { Correlación } \\
\text { c1-c2 } \\
\end{array}$ & $\begin{array}{c}\text { Correlación } \\
\text { c1 - < Dv }>\end{array}$ \\
\hline \multirow{2}{*}{$\begin{array}{c}\text { Velocidad de } \\
\text { formación de } \\
\text { capa }\end{array}$} & $\%<6 \mu \mathrm{m}$ & -0.655 & \multirow{2}{*}{0.440} \\
\hline & $\%<2 \mu \mathrm{m}$ & -0.651 & \\
\hline \multirow{2}{*}{ Viscosidad } & $\% \mathrm{MgO}$ & 0.689 & \multirow{2}{*}{-0.524} \\
\hline & $\%<2 \mu \mathrm{m}$ & 0.623 & \\
\hline \multirow{2}{*}{ Contracción } & $\% \mathrm{MgO}$ & 0.878 & \multirow{2}{*}{-0.637} \\
\hline & $\%<2 \mu \mathrm{m}$ & 0.690 & \\
\hline \multirow{2}{*}{$\begin{array}{l}\text { Densidad } \\
\text { aparente }\end{array}$} & $\%<6 \mu \mathrm{m}$ & 0.891 & \multirow{2}{*}{-0.732} \\
\hline & $\%<2 \mu \mathrm{m}$ & 0.875 & \\
\hline \multirow{2}{*}{$\begin{array}{l}\text { Absorción de } \\
\text { agua }\end{array}$} & $\%<2 \mu \mathrm{m}$ & -0.922 & \multirow{2}{*}{0.813} \\
\hline & $\%<6 \mu \mathrm{m}$ & -0.902 & \\
\hline
\end{tabular}

Viscosidad y contracción presentan las mayores correlaciones con $\mathrm{MgO}$ y porcentaje de fracción inferior a 2 $\mu \mathrm{m}$; la influencia del $\mathrm{MgO}$ puede relacionarse con que vaya asociado a láminas de minerales micáceos o a presencia de láminas de esmectitas, o incluso a esmectitas, como se ha puesto de manifiesto en la muestra k1. Norton (22) señala la mayor fluidez de vidrios con $\mathrm{Mg}^{2+}$, y alude al efecto fundente de impurezas micáceas dispersas que favorecen la difusión de álcalis en pastas triaxiales.

$<$ Dv $>$ presenta correlaciones significativas con las características tecnológicas; negativas con la densidad aparente y la contracción, y positiva con la absorción de agua..

\section{CONCLUSIONES}

Los materiales estudiados, actualmente utilizados en pastas de colaje (por ejemplo para porcelanas triaxiales) muestran una notable semejanza composicional.

Se ha apreciado una correlación negativa significativa entre el tamaño de cristalito y la densidad aparente y contracción a $1200{ }^{\circ} \mathrm{C}$ y positiva entre el tamaño de cristalito y la absorción de agua a la misma temperatura.

Estos hechos pueden interpretarse en el sentido de que en un conjunto de muestras de bastante similitud composicional y en cuya fracción fina predomina netamente la caolinita, la gresificación a $1200{ }^{\circ} \mathrm{C}$ es mayor en muestras cuya caolinita presenta menor tamaño de cristalito, y viceversa.

De ello se deriva la utilidad del análisis microestructural de la caolinita en la evaluación de su influencia en las propiedades del producto cocido.

\section{BIBLIOGRAFÍA}

1. J. Konta; "Clay and man: Clay raw materials in the service of man" Appl. Clay Sci. 10, 275-335 (1995).

2. D.G. Williams, C.L. Garey; "Crystal imperfections with regard to direction in kaolinite mineral" Clays Clay Miner. 22, 117-125 (1974).

3. G.K. Williamson, W.H. Hall; "X-ray line broadening from filed aluminium and wolfram" Acta Metallurgica 1, 22-31 (1953).

4. B.E. Warren, B.L. Averbach; "The effect of cold work distorsion on X-ray patterns" J. Appl. Phys. 21, 595-599 (1950).

5. J.M. Amigó, J. Bastida, A. Sanz, M. Signes y J. Serrano; “Crystallinity of Lower Cretaceous kaolinites of Teruel (Spain)" Appl. Clay Sci. 9, 51-69 (1994).

6. P. Aparicio, E. Galán; “Mineralogical interference on kaolinite crystallinity index measurements" Clays Clay Miner. 47, 1, 12-27 (1999) 
7. S. Guggenheim, D.C. Bain, F. Bergaya, M.F. Brigatti, V.A. Drits, D. Eberl, M.L. Formoso, E. Galán, R.J. Merriman, D.R. Peacor, H. Staniek, T. Watanabe. Report of the AIPEA Nomenclature Commitee for 2001: “Order, disorder and crystallinity in phyllosilicates and the use of the "Crystallinity index" Clay Miner. 37, 389-393 (2002)

8. J.V. Clausell; “Análisis microestructural de caolinitas y génesis de caolines en el Macizo Ibérico" Cadernos do Laboratorio Xeoloxico de Laxe (26) 1199 (2001)

9. D. Eberl, V. Drits, J. Srodon, R. Nüesch; “MudMaster: a program for calculating crystallite size distributions ans strain from the shapes of X-ray diffraction peaks" U.S. Geological Survey Open File Report, 96-171 (1996)

10 E. Galán, J. Espinosa; “El caolín en España” Sociedad Española de Cerámica y Vidrio. Madrid,1974. 224 pp.

11 AENOR, Norma Española UNE-En ISO 10545-3, “Baldosas cerámicas Parte 3: Determinación de la absorción de agua, de la porosidad abierta, de la densidad relativa aparente y de la densidad aparente".

12 J.L.Amorós, E. Sánchez, J. García-Ten, V. Sanz, M. Monzó “Manual para el control de la calidad de materias primas arcillosas". Instituto de Tecnología Cerámica-AICE, 1998. Castellón, 178 pp.
13. C. Warshall, R. Roy, "Classification and scheme for the identification of layer silicates". Geol. Soc. Am. Bull. 72, 1455-1492 (1961)

14. B.L. Davis, D. Smith, "Table of Experimental Reference Intensity Ratios" Powder Diffr. 3, 201-205 (1989)

15. B.L. Davis, D. Smith, "Table of Experimental Reference Intensity Ratios. Table n ${ }^{\circ}$ " Powder Diffr. 4, 206-209 (1989)

16. J.I. Langford, "A rapid method for analysing breadths of diffraction and spectral lines using the Voigt function" J. Appl. Crystallogr. 11, 50-55 (1978)

17. T. De Keijser, J.I. Langford, E.J. Mittemeijer, A.B.P. Vogels, "Use of the Voigt function in a single-line method for the analysis of X-ray diffracction line broadening" J. Appl. Crystallogr. 15, 308-314 (1982)

18 E. Gippini, "Pastas Cerámicas" Inst. Eduardo Torroja de la Construcción y el Cemento. pp 234-239. Madrid (1979)

19 A. Caballero, S. de Aza, "Efectos del grado de molienda sobre la microestructura y propiedades de las bauxitas refractarias" Bol. Soc. Esp. Ceram. V. 27, 2, 81-88 (1988)

20 I. Ferrer Franco, I. Ferrer Olmos, "Influencia de la finura del cuarzo en las porcelanas feldespáticas” Bol. Soc. Esp. Ceram. V. 30, 1, $23-27$ (1991)

21 "Tecnología cerámica aplicada". Vol 1. Ed. Faenza Edittrice (2004)

22 F.H. Norton, "Cerámica fina, tecnología y aplicaciones" Ed. Omega (Barcelona), 505 pp. (1988)

Recibido: 08.01 .04

Aceptado: 17.05 .05 